\title{
High Expression of RAGE and HMGB1 in Lung Tissue of Decedents with COVID-19 and Diabetes
}

\author{
Yared Tekabe, Anjali Saqi, Geping Zhang, Monica Goldklang and Lynne L Johnson* \\ Columbia University Medical Center, New York, USA
}

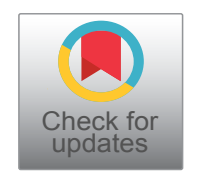

*Corresponding author: Lynne L Johnson, MD, Columbia University Medical Center, New York, USA, Tel: 212-305-7594, Fax: 212-305-4648

\begin{abstract}
Background: Serum biomarker studies on patients with severe COVID-19 lung disease indicate that increased ligands for Receptor for Advanced Glycation End-products (RAGE) and diabetes are risk factors for severe acute respiratory distress syndrome (ARDS).

Methods: To investigate in situ RAGE expression in coronavirus disease-19 (COVID-19) ARDS, we obtained lung tissue from autopsies performed on COVID-19 decedents with clinical ARDS and corresponding pathology showing diffuse alveolar damage (DAD) both with and without diabetes. These were compared with a cohort of pre-pandemic, COVID-19 (-) decedents with ARDS/DAD without diabetes. Quantitative immunohistochemical (IHC) staining for RAGE and immunofluorescence (IF) staining for RAGE and high mobility group box 1 (HMGB1), a damage associated molecular pathway ligand for RAGE, were performed on 3 cohorts: 5 diabetic and 5 non-diabetic COVID-19 decedents and 5 ARDS/DAD decedents without COVID-19; normal lung biopsy tissue served as control. RAGE staining was first counted for each decedent and the overall averages for each cohort were statistically compared.
\end{abstract}

Results: RAGE staining was significantly higher in COVID-19 decedents with diabetes compared to decedents without diabetes, and there was no significant difference between non-diabetics with and without COVID-19. IF staining for HMGB1was higher in COVID-19 decedents with diabetes and co-localized with RAGE.

Conclusions: These findings in a small group of carefully selected decedents who died from COVID-19 ARDS suggest that RAGE expression is significantly increased in a setting of COVID-19 and diabetes and likely plays a role in fatal outcome.

\begin{abstract}
Abbreviations
AEC: Alveolar Epithelial Cells; AGES: Advanced Glycation Endproducts; ALI: Acute Lung Injury; ARDS: Acute Respiratory Distress Syndrome; DAD: Diffuse Alveolar Damage; DAMPS: Damage Associated Molecular Pathways; HMGB1: High Mobility Group Box 1; IHC: Immunohistochemistry; PAMPS: Pathogen Associated Molecular Pathways; PCR: Polymerase Chain Reaction; RAGE: Receptor for Advance Glycation Endproducts; SARS: Severe Acute Respiratory Syndrome; SPC: Surfactant Protein C; TLR: Toll Like Receptors
\end{abstract}

\section{Introduction}

Older patients with obesity and/or chronic diseases such as hypertension, underlying chronic lung disease, and diabetes are at highest risk for developing severe complications of COVID-19 lung disease and dying from acute respiratory distress syndrome (ARDS) [1-3]. In diabetes, high circulating levels of glucose bind proteins in a non-enzymatic reaction producing Advanced Glycation Endproducts (AGEs). High circulating levels of AGEs and other ligands bind a receptor (Receptor for Advanced Glycation Endproducts, RAGE) generating more receptors and ligands in a positive feedback loop [4]. RAGE ligands S100 and high mobility group box 1 (HMGB1) mediate pathogen-associated molecular patterns (PAMPS) and Damage Associated Molecular Patterns (DAMPS) that perpetuate and amplify inflammatory responses in the lung to infectious agents [5-8]. These serum biomarkers have been associated with increased mortality in COVID-19 patients $[9,10]$.

Citation: Tekabe Y, Saqi A, Zhang G, Goldklang M, Johnson LL (2022) Comparison High Expression of RAGE and HMGB1 in Lung Tissue of Decedents with COVID-19 and Diabetes. Int J Pathol Clin Res 8:123. doi.org/10.23937/2469-5807/1510123

Accepted: February 15, 2022: Published: February 17, 2022

Copyright: (C) 2022 Tekabe $\mathrm{Y}$, et al. This is an open-access article distributed under the terms of the Creative Commons Attribution License, which permits unrestricted use, distribution, and reproduction in any medium, provided the original author and source are credited. 
To further investigate the role of RAGE expression in COVID-19 lung disease, we evaluated lung tissues from patients with SARS-CoV-2 confirmed by polymerase chain reaction (PCR) who died with ARDS during the height of the pandemic in NYC in spring 2020. We performed immunohistochemical (IHC) and dual immunofluorescence (IF) staining of lung tissue sections from selected patients to test our hypothesis that RAGE is overexpressed among diabetics with COVID-19 ARDS.

\section{Methods}

\section{Case selection}

Autopsy reports from patients with either premortem or postmortem RT-PCR-confirmed SARS-CoV-2 were reviewed. COVID-19 lung tissue from decedents with ARDS and histologically confirmed diffuse alveolar damage (DAD) from both diabetic and non-diabetic patients were identified. For comparison, pre-pandemic COVID-19 cases from decedents without viral illness and whose cause of death was ARDS secondary to sepsis outside the lung were selected. Tissue from selected decedents was provided by the Columbia University Medical Center COVID-19 Molecular Pathology Shared Resource. A surgical specimen from a pneumothorax case served as a normal control. All tissue samples were deidentified.

\section{Histology, IHC and IF}

Tissue sections were analyzed from ARDS/DAD SARS-CoV-2 positive cases with and without diabetes against ARDS/DAD SARS-CoV-2 negative cases without diabetes. Sections from specimens removed from a patient with pneumothorax and no known other lung pathology were stained but not quantified. Serial tissue sections were stained for hematoxylin and eosin (H\&E) and for RAGE IHC. The tissue sections were incubated overnight with humanized anti-RAGE antibody (50 $\mu \mathrm{g} / \mathrm{ml}$ ) followed by incubation for 30 min with HRPconjugated biotinylated secondary antibody (1:200). Dual IF staining was performed for RAGE with red fluorescence, and with green fluorescence for cell type: Macrophages, type I alveolar cells (aquaporin 5), type II alveolar cells surfactant protein C (SPC), and for HMGB1. Merging produced yellow fluorescence to identify colocalization.

\section{Alveolar cell types}

Dual fluorescence staining was performed to identify type I and type II alveolar epithelial cells (AEC). The two different types of cells composing the alveolar epithelial lining were identified by selective staining. Alveolar type I epithelial cells comprise about $80 \%$ of the alveolar surface area and are primarily responsible for gas exchange. Meanwhile, alveolar type II epithelial cells play the critical roles of producing surfactant, moving water out of the airspaces, and regenerating alveolar epithelium.

\section{Quantitative staining for RAGE}

Morphometric and quantitative analyses were performed using a Nikon Eclipse 50i confocal microscope (Nikon, Tokyo, Japan) and Image-Pro Plus software (Media Cybernetics Inc., Silver Spring, MD).

\section{Statistics}

Comparisons of averages among the three cohorts (COVID-19 with and without diabetes and non-COVID-19
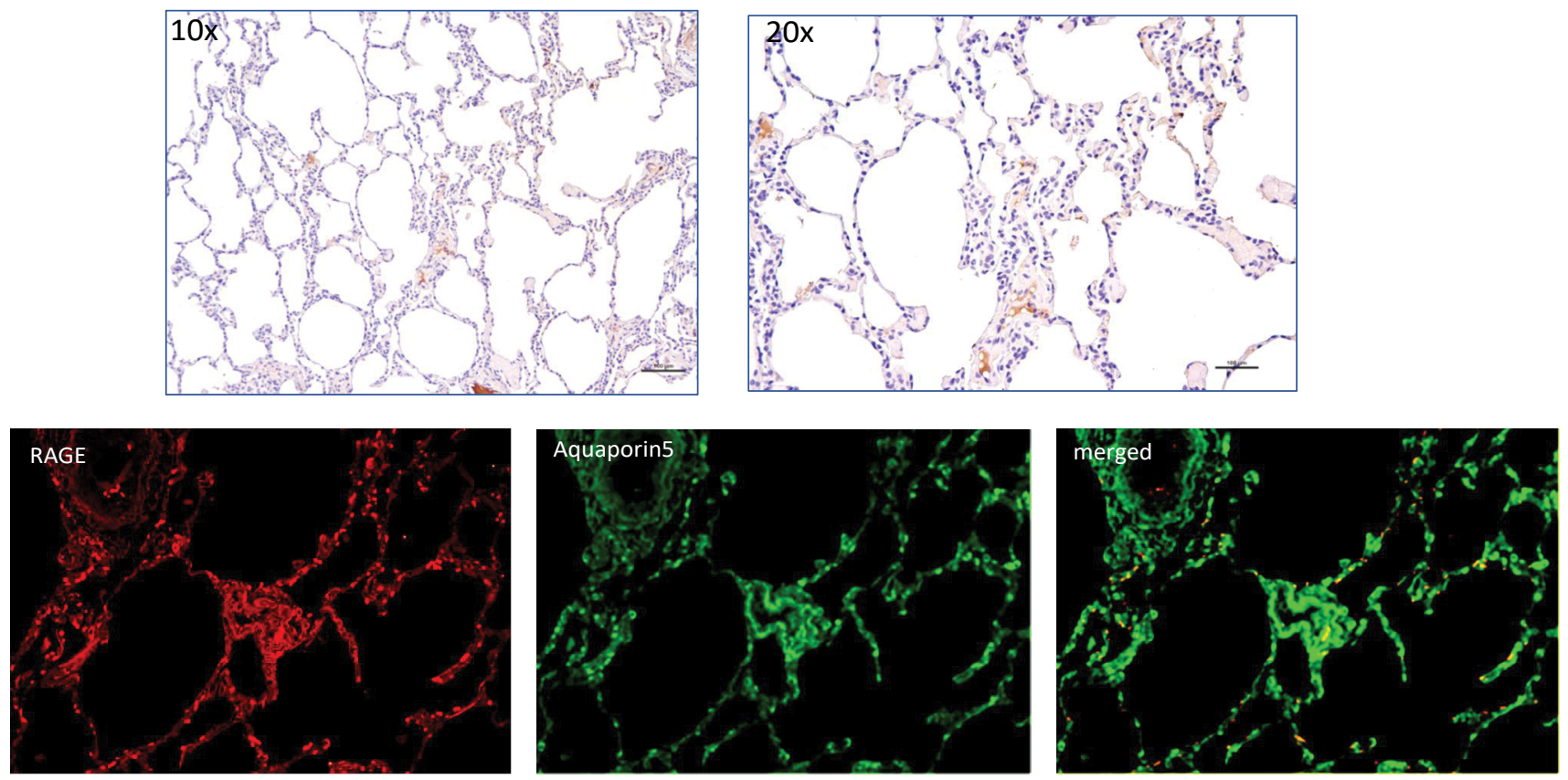

Figure 1: Top row shows H\&E staining for RAGE at 10X and 20X from normal lung section taken from a subject with pneumothorax. The lower row shows IF staining for RAGE (red) on left, Aquaporin5 in middle, and merged IF on the right with yellow/ orange showing co-localization of IF staining for RAGE and alveolar cell type 1. 
without diabetes) with equal variance were made using two sample t tests. A $P$ value $<0.05$ was considered significant.

\section{Results}

\section{Staining with RAGE in control lung tissue}

RAGE expression by IHC was detected but in low levels in lung tissue from a surgical specimen from otherwise healthy individual with pneumothoraxselected as a negative control (Figure 1). Dual IF showed co-localization of RAGE and type I alveolar epithelial cells (Figure 1).

\section{Staining for RAGE in lung tissue from decedents with ARDS}

Stained sections from a non-diabetic patient who tested positive for SARS-CoV-2 on admission and who died with ARDS are shown in Figure 2. The H\&E sections from an area of organizing DAD (Figure 2a) and corresponding section with RAGE IHC show brown chromogen positive staining predominantly localized to intra-alveolar spaces containing predominantly macrophages (Figure 2b). Dual IF staining for RAGE and Aquaporin 5 (type I AEC) and surfactant protein C (SPC) showed co-localization to both cell types but with greater co-staining to type I AEC (Figure 2c). The lung tissue at the time of autopsy tested negative for SARS-CoV-2, and there was no evidence for bacterial pneumonia, which suggests that the progressive lung damage during the hospital course was due at least in part to immunological assault. Similar staining was seen in the remaining 4 decedents with SARS-CoV-2 and no diabetes.

Stained tissue sections from a diabetic patient who tested positive for SARS-CoV-2 and died with ARDS are shown in Figure 3 . The H\&E sections show dense infiltrates completely obliterating air spaces (Figure 3a). RAGE IHC (Figure 3b) shows more extensive brown chromogen positive staining compared to RAGE staining seen in non-diabetic patient shown in Figure 2b. Some of the staining appears to be in the extracellular space, which may represent soluble receptor (sRAGE) cleaved from the cell membrane. Dual IF staining for RAGE and Aquaporin 5 (type I AEC) and surfactant protein C (SPC) showed co-localization to both cell types with qualitatively greater co-localized staining for type I AECS and greater staining for both AEC type I and II than seen in non-diabetic patient (Figure 3c). A similar pattern was evident in the remaining 4 decedents with SARS-CoV-2 and diabetes.

Stained tissue sections from a non-diabetic patient negative for SARS-CoV-2 with ARDS secondary to sepsis arising from a non-pulmonary source are shown in Figure 4. The H\&E stained sections show scant hyaline membranes and inflammation (Figure 4a). As shown in Figure $4 b$, there is RAGE staining. Quantitatively it appears much less extensive than seen in the tissue from the SARS-CoV-2 positive decedent with diabetes and similar in extent to the non-diabetic SARS-CoV-2 positive tissue. Dual fluorescence showed localization to both type I and type II alveolar epithelial cells (Figure $4 c)$.

\section{Dual IF staining for HMGB1 and macrophages}

Figure 5 shows dual IF staining for RAGE and HMGB1 in the non-diabetic patient who tested positive for SARSCoV-2 (Figure 2) and the diabetic patient who tested positive for SARS-CoV-2 (Figure 3). The merged stains show co-localization of receptor and ligand with greater merged fluorescence signal in the diabetic patient. These findings suggest a role for the damage associated molecular pathway (DAMP) activation by HMGB1in an overactive immune response contributing to disease severity in COVID-19.

\section{Quantitative RAGE staining}

Quantitative analysis of RAGE by IHC staining was performed on 15 lung sections (a single section from each decedent in all three cohorts). The average \% a
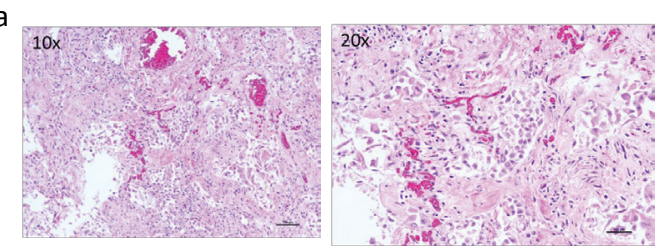

b
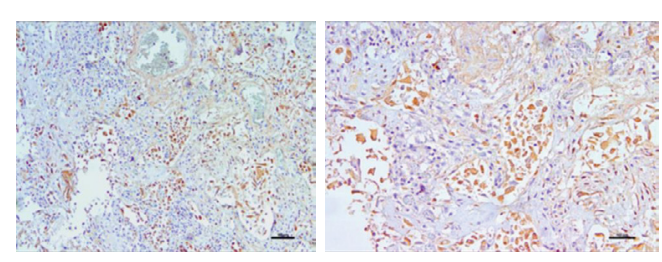

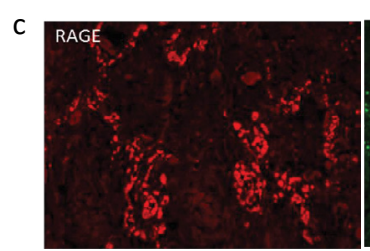

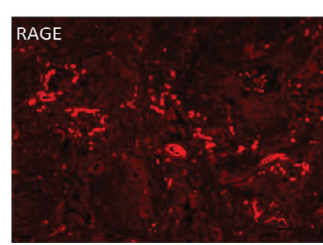

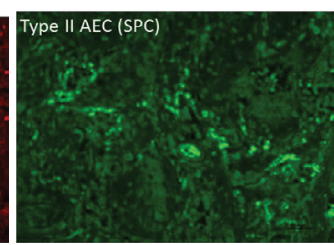

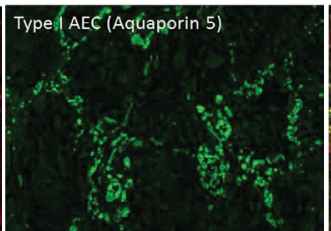
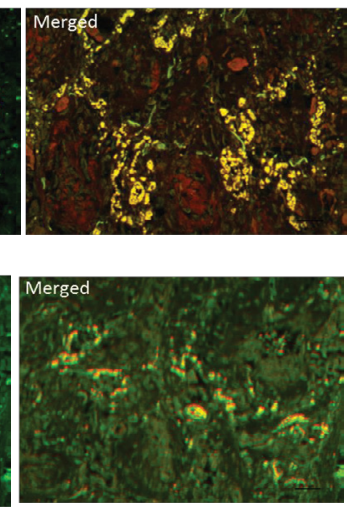

Figure 2: Left panel shows H\&E (top row) (a) and IHC staining for RAGE (brown chromogen) (bottom row) (b) on lung sections from non-diabetic decedent positive for SARS-CoV-2 who died with ARDS. There are extensive intra-alveolar infiltrates with cells that stain positive for RAGE. Right panel c shows in top row, IF for RAGE (red) and type I AEC (Aquaporin5) and on bottom row RAGE and type 2 AEC (surfactant producing cells-SPC) (green) with co-localization predominantly to type I AEC. 

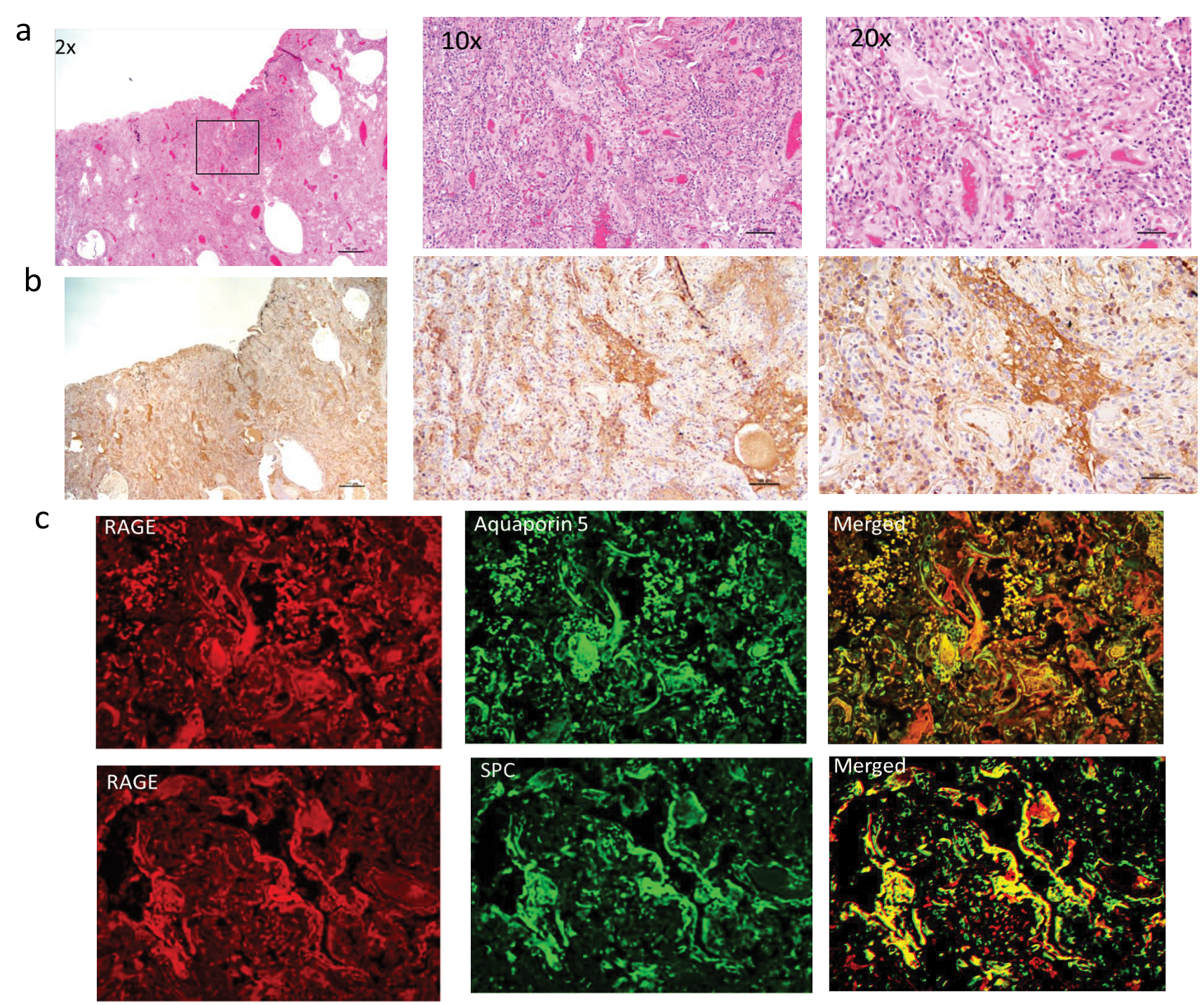

Figure 3: Row a shows H\&E and row b shows IHC staining for RAGE (brown chromogen) on lung sections from diabetic decedent positive for SARS-CoV-2 who died with ARDS. There is organizing DAD. The RAGE staining (brown chromogen) is more extensive than shown in non-diabetic patient in figure 2. Rows c and d show IF for RAGE (red) and alveolar epithelial cell type I (Aquaporin5) and type 2 (surfactant producing cells-SPC) (green) with co-localization to both types of alveolar endothelial cells.
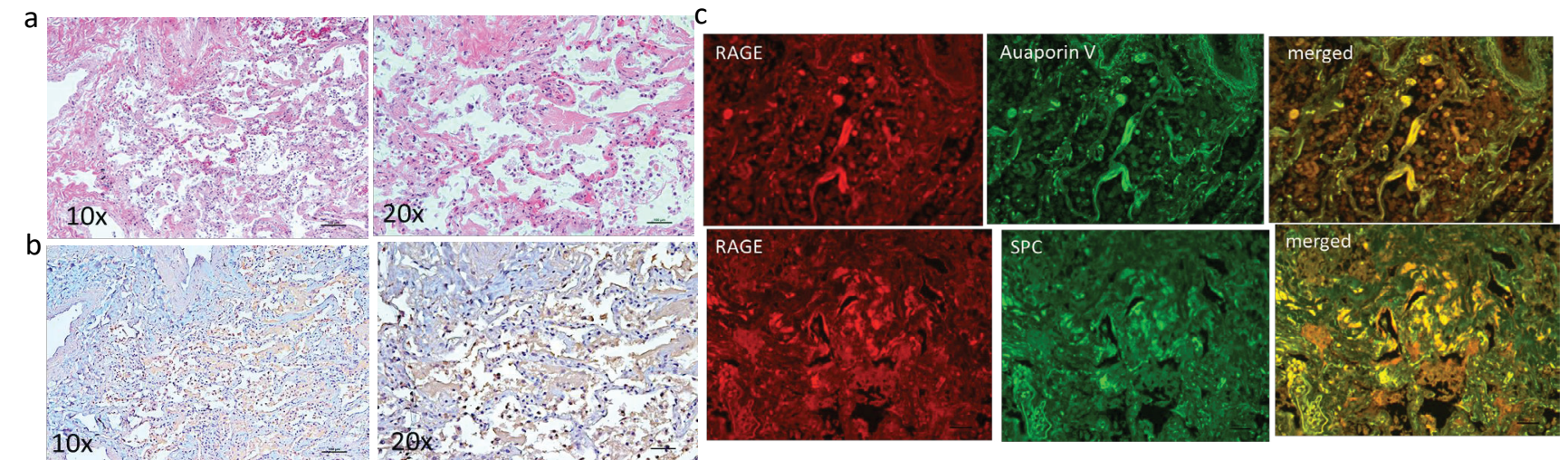

Figure 4: Left panel shows H\&E (top row) (a) and IHC staining for RAGE (brown chromogen) (bottom row) (b) on lung sections from non-diabetic decedent negative for SARS-CoV-2 who died with ARDS secondary to sepsis from a non-pulmonary source. There are intra-alveolar infiltrates with cells that stain positive for RAGE as seen on sections shown in row $b$. Right panel c shows in top row, IF for RAGE (red) and type I AEC (Aquaporin5) and on bottom row RAGE and type 2 AEC (surfactant producing cells-SPC) (green) with co-localization predominantly to type I AEC. 

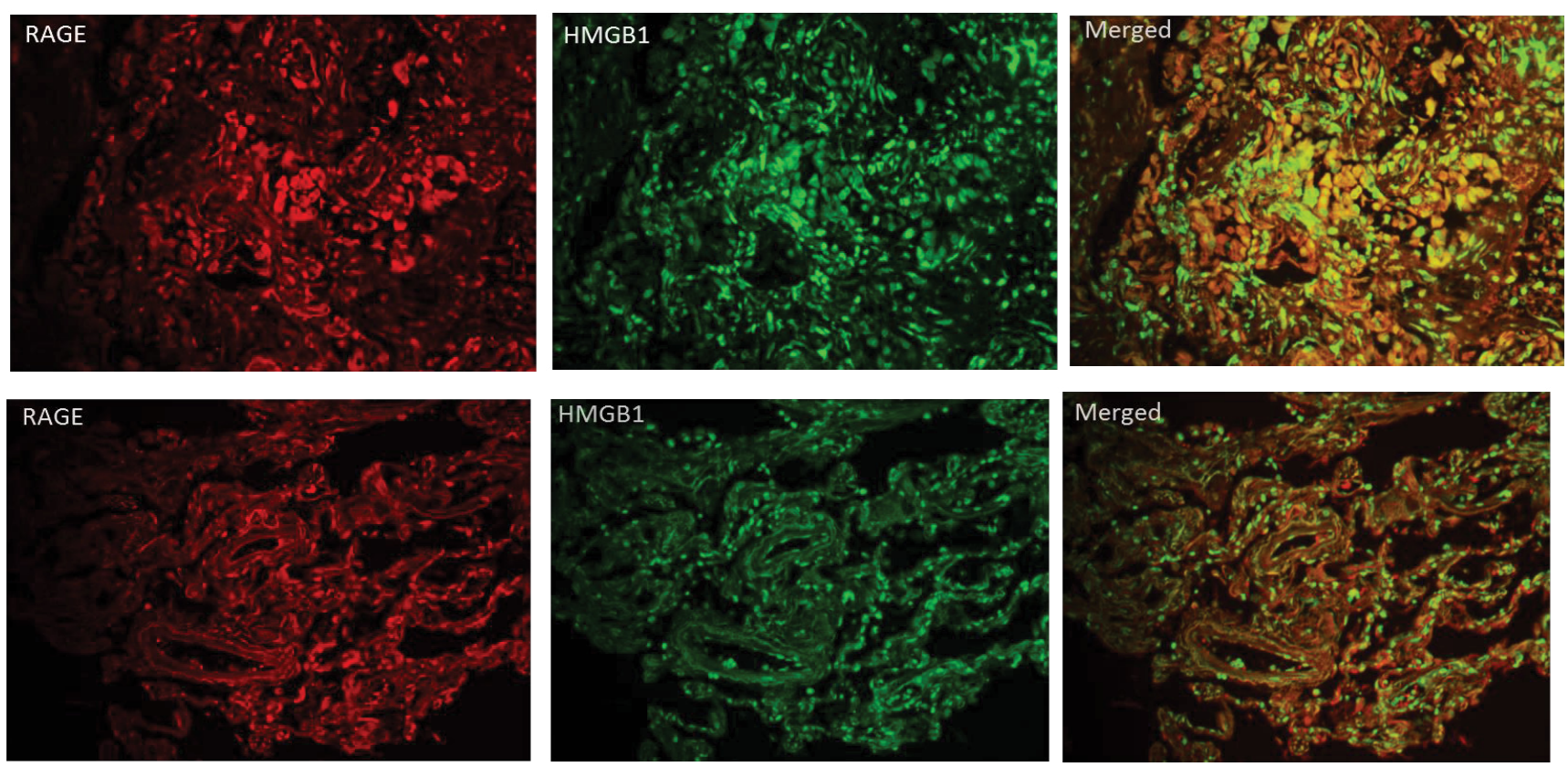

Figure 5: IF staining on lung sections from a diabetic decedent with COVID-19 ARDS (top row) and from a non-diabetic decedent with COVID-19 ARDS (bottom row). IF for RAGE (red) is on left, for HMGB1 (green) in middle and co-localization (yellow) on right. Qualitatively there is greater HMGB1/RAGE IF in the lung from the diabetic decedent.

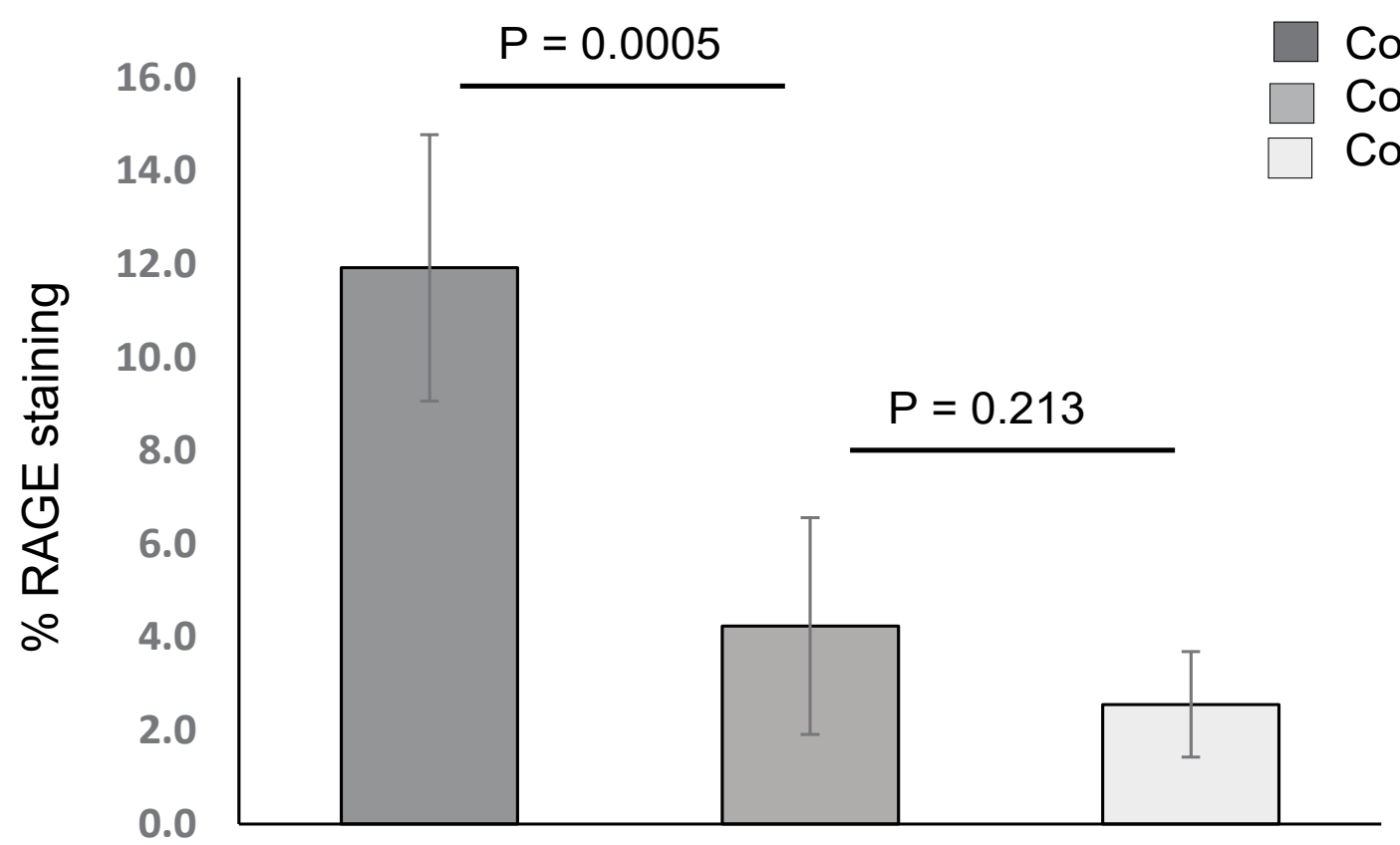

Figure 6: Bar graph showing mean \pm SD for quantitative RAGE IHC on lung tissue from five SARS-CoV-2 + diabetic decedents with ARDS (dark gray bar), five SARS-CoV-2 + non-diabetic decedents with ARDS (medium gray bar), and 5 non-diabetic SARS-CoV-2 negative decedents with ARDS (light gray bar). There was statistically significant greater staining in the lung tissue from diabetic decedents than the non-diabetic and no statistically significant difference between the SARS-CoV-2 + non-diabetic ARDS decedents and the SARS-CoV-1 negative non-diabetic ARDS decedents.

area staining for the brown chromogen (RAGE) per $100 x$ field on the lung tissue sections from SARS-CoV-2 positive decedents with diabetes was $11.9 \pm 2.3 \%$ and $4.2 \pm 2.3 \%$ for lung tissue from COVID-19 decedents without diabetes $(P=0.0005)$ (Figure 6). Average \% RAGE staining from 5 decedents who died with ARDS and sepsis from non-lung source was $2.56 \pm 1.13 \%$ which was not statistically different from the average values for the lung tissue sections from the non-diabetic COVID-19 ARDS decedents $(P=0.213)$.

\section{Discussion}

To date all reports implicating HMGB1 in COVID-19 have been serum biomarker studies. This study is the 
first to perform RAGE IHC and RAGE/HMGB1IF on lung sections from decedents with COVID-19 ARDS and corroborate results of previously reported serum biomarkers studies. In this report, we show that lung tissue from decedents with confirmed severe COVID-19 ARDS have increased RAGE expression, and it is greater in diabetics compared to non-diabetics. Dual IF staining showed higher expression of RAGE in type II alveolar epithelial cells in diabetic compared to non-diabetic cases. In lung tissue from patients with ARDS alone (without COVID-19), there is also RAGE staining which is expected given the role of RAGE in mediating the inflammatory response, but the level of expression is much lower than in the SARS-CoV-2 tissue from diabetics. There was high IF signal for DAMPS ligand HMGB1 in lung tissue of COVID-19 cases, with qualitatively greater staining in diabetic compared to non-diabetic case and co-localized with RAGE. This pilot data supports the premise that RAGE plays a role in the pathology of ARDS/DAD in COVID-19and it may be mediated in part through DAMPS activated by HMGB1 ligand binding.

In healthy individuals, RAGE expression is nondetectable to low in all organs of the body except the lungs, where constitutive expression of RAGE is higher than other body organs in many species including humans $[11,12]$. Because immune responses to viral illnesses originate in the lungs suggests that RAGE may play a role in this response. In normal human lung tissue, RAGE immunoreactivity is found in bronchiolar epithelia, type II alveolar pneumocytes, and alveolar macrophages as well as the endothelium of larger arteries [10]. Mouse models of acute lung injury (ALI) including our own data show that injury induces RAGE expression and increases in RAGE ligands [13]. The binding of receptor and ligands activate pathways of inflammation and tissue damage $[4,13]$. RAGE inhibition by blocking RAGE/ligand binding will reduce both receptor and ligand levels in tissue and reduce lung injury [14].

In a study of 40 postmortem examinations in COVID-19 patients, 2 pathological phenotypes were identified [15]. The major pulmonary pattern observed was $\mathrm{ALI}$ in $73 \%$ of cases and non-ALI in the minority of cases. The pathology in ALI was described as hyaline membranes consistent with DAD, which correlated with the clinical diagnosis of ARDS. This pathology was present in the non-diabetic and diabetic patients dying from COVID-19 with positive immune staining for RAGE.

RAGE is a multi-ligand receptor and member of the immunoglobulin super-family of cell surface receptors [16]. The full receptor consists of 5 domains: the cytosolic domain, which is responsible for signal transduction, the transmembrane domain which anchors the receptor in the cell membrane, the variable domain (V) which binds the RAGE ligands, and two constant (C) domains [16]. It functions as transmembrane pattern recognition receptor that binds multiple ligands including DAMPS and PAMPS released by damaged cells undergoing cell death $[17,18]$. One of the DAMPS passively released by dying cells is HMGB1, which is normally located intracellularly acting as a DNA chaperone to facilitate access to transcriptional factors to target specific genes [17]. Research suggests that this local release and binding does not directly lead to proinflammatory intracellular signaling but rather that RAGE enables transport of HMGB1-partner DNA and RNA molecule complexes to bind cognate cytoplasmic receptors that lead to overwhelming inflammation [17].

In viral and bacterial disease, both soluble RAGE (extracellular component of receptor cleaved at cell membrane), and HMGB1 are released into the circulation and have been shown to be markers of poor prognosis [7-9]. In viral infections, as cells die, they release HMBG1 either passively from necrotic cells or actively by secretion [18]. The HMGB1 released promotes the inflammatory response by binding to cell membrane receptors specifically TLR2/4 and RAGE [18]. HMGB1/RAGE binding leads to the activation of the NF$\mathrm{KB}$ and MAPK pathways, while HMGB1 interactions with TLR2 and TLR4 mediate immune activation contributing to cytokine storm $[17,18]$. With these known properties, RAGE and HMGB1 have been implicated in disease severity in bacterial and viral infections and recently in COVID-19 [7-9].

\section{Summary}

RAGE is a transmembrane receptor that is highly expressed in lung tissue of experimental models of ALI and circulating SRAGE and DAMPS such as HMGB1 associated with increased severity of viral and bacterial pneumonia. We performed IHC and dual IF staining of autopsy lung tissue from selected decedents positive for SARS-CoV-2 on hospital admission who died with ARDS. We found increased expression of RAGE in tissue from both diabetic and non-diabetic decedents, with statistically significant higher expression of RAGE and HMGB1 in diabetic lung tissue. Although a small sample, these findings support the premise that RAGE and DAMPS contribute to COVID-19 lung disease and cytokine storm. While all cases in this study are from autopsies and resulted in fatal outcomes, increased expression of RAGE/HMGB1 among diabetics portends greater risk.

\section{Sources of Support}

This research was funded in part through the NIH/ $\mathrm{NCl}$ Cancer Center Support Grant P30CA013696 and has utilized the Molecular Pathology Shared Resource.

\section{Author Contributions}

All authors made equal contributions.

\section{References}

1. Argenziano MG, Bruce SL, Slater CL, Tiao JE, Baldwin 
MR, et al. (2020) Characterization and clinical course of 1000 patients with coronavirus disease 2019 in New York: Retrospective case series. BMJ 369: m1996.

2. Elamari S, Motaib I, Zbiri S, Elaidaoui K, Chadli A, et al. (2020) Characteristics and outcomes of diabetic patients infected by the SARS-CoV-2. Pan Afr Med J 37: 32.

3. Scheen AJ, Marre M, Thivolet C (2020) Prognostic factor in patients with diabetes hospitalized for COVID-19: Findings from the CORONADO study and other recent reports. Diabetes Metab 46: 265-271.

4. Ramasamy R, Yan SF, Schmidt AM (2012) Advanced glycation endproducts: From precursors to RAGE: Round and round we go. Amino Acids 42: 1151-1161.

5. Nakamura T, Sato E, Fujiwara N, Kawagoe Y, Maeda S, et al. (2011) Increased levels of soluble receptor for advanced glycation end products (sRAGE) and high mobility group box 1 (HMGB1) are associated with death in patients with acute respiratory distress syndrome. Clin Biochem 44: 601604.

6. Narvaez-Rivera RM, Rendon A, Salinas-Carmona MC, Rosas-Taraco AG (2012) Soluble RAGE as a severity marker in community acquired pneumonia associated sepsis. BMC Infect Dis 12: 15.

7. Van Zoelen MAD, Van der Sluijs KF, Achouiti A, Florquin S, Braun-Pater JM, et al. (2009) Receptor for advanced glycation end products is detrimental during influenza $A$ virus pneumonia. Virology 391: 265-273.

8. Qu Y, Zhan Y, Yang S, Ren S, Qiu X, et al. (2018) Newcastle disease virus infection triggers HMGB1 release to promote the inflammation response. Virology 525: 19-31.

9. Abers MS, Delmonte OM, Ricotta EE, Fintzi J, Fink DL, et al. (2020) An immune-based biomarker signature is associated with mortality in COVID-19 patients. JCI Insight 6: e144455.
10. Chen L, Long X, Xu Q, Tan J, Wang G, et al. (2020) Elevated serum levels of S100A/A9 and HMGB1 at hospital admission are correlated with inferior clinical outcomes in COVID-19 patients. Cell Mol Immunol 17: 992-994.

11. Mukherjee TK, Mukhopadhyay S, Hoidal JR (2008) Implication of receptor for advanced glycation end product (RAGE) in pulmonary health and pathophysiology. Respir Physiol Neurobiol 162: 210-215.

12. Buckley ST, Ehrhardt C (2010) The receptor for advanced glycation end-products and the lung. J Biomed Biotechnol 2010: 917108.

13. Su X, Looney MR, Gupta N, Matthay MA (2009) Receptor for advanced glycation end-products (RAGE) is an indicator of direct lung injury in models of experimental lung injury. Am J Physiol Lung Cell Mol Physiol 297: L1-L5.

14. Blondonnet R, Audard J, Belville C, Clairefond G, Lutz J, et al. (2017) RAGE inhibition reduces acute lung injury in mice. Sci Rep 7: 7208 .

15. De Michele S, Sun Y, Yilmaz MM, Katsyv I, Salvatore M, et al. (2020) Forty postmortem examinations in COVID-19 patient: Two distinct pathologic phenotypes and correlation with clinical and radiologic findings. Am J Clin Pathol 154: 748-760.

16. Bongarzone S, Savickas V, Luzi F, Gee AG (2017) Targeting the receptor for advanced glycation endproducts (RAGE): A medical chemistry perspective. J Med Chem 60: 7213-7232.

17. Yang $H$, Liu $H$, Zeng $Q$, Imperato $G H$, Addorisio ME, et al. (2019) Inhibition of HMGB1/RAGE- mediated endocytosis by HMGB1 antagonist box A, anti-HMGB1 antibodies, and cholinergic agonists suppresses inflammation. Mol Med 25: 13.

18. Andersson U, Ottestad W, Tracey KJ (2020) Extracellular HMGB1: A therapeutic target in severe pulmonary inflammation including COVID-19. Mol Med 26: 42. 